\title{
Time-resolved luminescence of semiconductor heterostructures in high magnetic fields
}

\author{
A.P. Heberle ${ }^{a}$, S. Haacke ${ }^{b}$, M. Oestreich ${ }^{a}$, M. Potemskib ${ }^{b}$ W.W. Rühle ${ }^{a} *$, J.C. Maan ${ }^{c}$, \\ K. Köhler ${ }^{\text {, }}$ G. Weimann ${ }^{\mathrm{e}}$, H.-J. Queisser ${ }^{\mathrm{a}}$ \\ ${ }^{a}$ Max-Planck-Institut für Festkörperforschung, Heisenbergstrasse 1, D-70506 Stuttgart, Germany

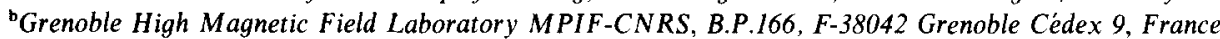 \\ ${ }^{\mathrm{c}}$ High Field Magnet Laboratory and Research Institute for Materials, University of Nijmegen, 6525 ED Nijmegen, The Netherlands \\ ${ }^{\mathrm{d}}$ Fraunhofer-Institut für Angewandte Festkörperphysik, Tullastrasse 72, D-79108 Freiburg, Germany

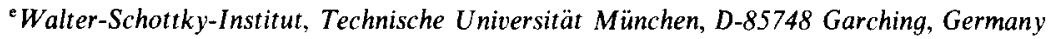

\begin{abstract}
We report on time-resolved luminescence in GaAs quantum wells in high magnetic fields up to $21 \mathrm{~T}$. Results on resonant tunneling and exciton relaxation including spin flip are presented.
\end{abstract}

\section{Introduction}

High magnetic fields and semiconducting materials exist in a harmonious symbiosis, resulting in fertile research and development [1]. Semiconductors provide essential advantages for investigations with very high fields. First, the adjustable and low charge carrier density allows for a strong reduction of carrier-carrier interactions, which strongly disturb the observation of magnetic field induced effects. Second, and this phenomenon may be even more significant, the usually very small effective electron mass of semiconductors causes strong, hence more easily measurable, effects, such as larger energy shifts and splittings.

A further important feature has recently become evident: semiconductors can be produced in the form of structures with reduced dimensionality [2]. New growth techniques, above all a highly controlled molecular beam epitaxy, nowadays yield layer structures of high crystal-

\footnotetext{
* Corresponding author.
}

line perfection, extending down to atomic dimensions. This availability, for example of quantum wells or quantum dots, ensures two vital consequences for the research with high magnetic fields. A new anisotropy of the materials arises; the growth direction, being perpendicular to the interface layers, now generates a novel degree of freedom for the orientation of the field with respect to this new crystalline axis. Variation of the field orientation hence opens new vistas for the study of electronic phenomena. The second feature resides in the manifold of new quantum confinement effects. Quantum wells, as an example, restrict the electronic wave functions and also transport to specific directions; the application of mag netic fields lifts the remaining degeneracies.

This paper describes some recent results involving semiconducting materials subjected to the high magnetic fields which are available at such designated laboratories as in the magnet laboratories of the Max-Planck-Institute and the CNRS in Grenoble, France. We first give a short general survey. Next, we describe recent experiments on resonant tunneling involving time-resolved spectroscopy and exciton relaxation including spin flip. 


\section{Survey}

The influence of a magnetic field on the energy spectrum of quantum wells depends on the direction of the magnetic field with respect to the growth axis. A magnetic field in growth direction causes formation of Landau levels and lateral magnetic confinement [3]. The Landau level degeneracy is removed by a magnetic field in the plane of the layers [4]. In both cases a magnetic field causes a splitting of spin degenerate electron levels and modifies exciton relaxation dynamics including spin flip as investigated by time-integrated luminescence [5].

Time-resolved optical experiments furnish direct insight into the carrier dynamics in magnetic fields. Quantum beats in solids were first observed between spin-split levels in magnetic fields [6]. Magnetic confinement of two-dimensional excitons was used to study thermalization between zero-dimensional levels [7]. We report on recent picosecond luminescence experiments with quantum wells in magnetic fields up to $21 \mathrm{~T}$ investigating the influence of a magnetic field perpendicular to the growth direction on the dynamics of resonant tunneling between quantum wells and the influence of a magnetic field in growth direction on electron spin dynamics.

\section{Experimental}

The samples are excited with pulses from a tunable Styryl 8 dye laser which is synchronously pumped by a frequency doubled Nd:YAG-laser at a repetition rate of $76 \mathrm{MHz}$. The carrier excitation density is below $10^{10} \mathrm{~cm}^{-2}$. The photoluminescence is dispersed by a $0.32 \mathrm{~m}$ spectrometer and detected by a two-dimensional streak camera with a spectral and temporal resolution of $0.5 \mathrm{~nm}$ and $15 \mathrm{ps}$, respectively. The samples are kept at a temperature of $2 \mathrm{~K}$ in a He bath cryostat, which is inserted in the bore of a $21 \mathrm{~T}$ Bitter magnet. Achromatic $\lambda / 4$ plates and linear polarizers are used to generate and detect light of circular polarization in the spin relaxation experiments.

Unfortunately, the large magnetic stray fields emanating from a $21 \mathrm{~T}$ Bitter magnet strongly deflect the electron beam inside the streak camera tube, and sufficient magnetic shielding is not feasible. However, the streak camera works reliably at a distance of more than $5 \mathrm{~m}$ from the magnet. Further reduction of the field effects is obtained if the electrons in the tube move parallel to the field. The final influence of the magnetic field on the camera is corrected by a field-dependent calibration of the time and wavelength axis.

\section{Tunneling}

Tunneling in semiconductor heterostructures is one of the most promising effects for eventual device applications of semiconductor quantum structures [8]. An asymmetric double quantum well (ADQW), which consists of a wide $\left(\mathrm{QW}_{\mathrm{w}}\right)$ and a narrow $\left(\mathrm{QW}_{\mathrm{n}}\right) \mathrm{GaAs}$ quantum well, separated by a thin $\mathrm{Al}_{x} \mathrm{Ga}_{1-x} \mathrm{As}$ barrier, permits direct access to the dynamics of nonresonant and resonant tunneling with the high time-resolution of optical techniques. Fig. 1 depicts the conduction (CB) and valence band (VB) of the ADQW in $z$-, the growth direction with the lowest electron and hole subband edges. The arrows show the channels for recombination and electronic tunneling. The luminescence decay rate $1 / \tau_{n}$ of $\mathrm{QW}_{\mathrm{n}}$ after excitation with a short laser pulse is the sum of the tunneling rate $1 / \tau_{\mathrm{T}}$ and the recombination rate $1 / \tau_{\mathrm{R}}$; the decay is thus dominated by tunneling if $\tau_{T}<\tau_{R}$. The "tunneling time $\tau_{\mathbf{T}}$ " is simply treated as an inverse rate $\tau_{\mathrm{T}}^{-1}$.

Resonant tunneling of electrons from $\mathrm{QW}_{\mathrm{n}}$ to $\mathrm{QW}_{\mathrm{w}}$ takes place, whenever the lowest subband of $Q W_{n}$ and a subband of $Q W_{w}$ are shifted to the same energy by an external electric field in $z$-direction. A strong resonant decrease of $\tau_{\mathrm{T}}$ was observed in a variety of experiments [9-12]. The resonance condition is not sensitive on the in-plane dispersion of the subbands, since resonance is achieved simultaneously for all wave vectors, if nonparabolicity is neglected.

In this paper we consider a different type of tunneling resonance induced by a magnetic field $B_{\perp}$ in $x$-direction. The field $B_{\perp}$ influences the lowest subbands $|w 1\rangle$ and $|n 1\rangle$ in $\mathrm{QW}_{\mathrm{w}}$ and $\mathrm{QW}$ in a twofold way: first, the band edge energies $E_{w 1}$ and $E_{n 1}$ experience a positive diamagnetic shift, an effect which is negligible in our experiment; second, the dispersion $E\left(k_{y}\right)$ of $|n 1\rangle$ is shifted relative to that of $|w 1\rangle$ by an amount $\Delta k_{y}=e \Delta z B_{x} / \hbar$, $\Delta z=\langle w 1|z| w 1\rangle-\langle n 1|z| n 1\rangle$ being the distance which the electrons tunnel [13]. While the diamagnetic shift is

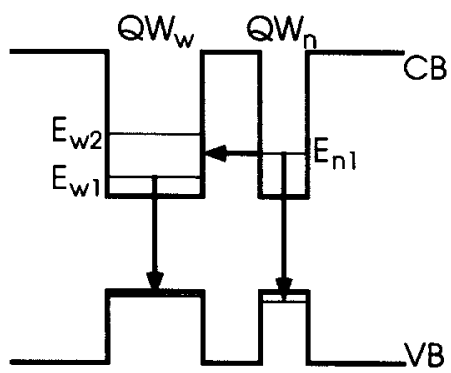

Fig. 1. Conduction (CB) and valence band (VB) of an ADQW in the growth direction with the lowest electron and hole levels. The arrows indicate recombination and electronic tunneling. 
a purely quantum mechanical effect, the shift of dispersion can also be understood semiclassically: an electron tunnels perpendicular to the magnetic field and receives therefore a momentum $\hbar \Delta k_{y}$ due to the integrated Lorentz force acting during the tunneling transfer. The left inset of Fig. 2 shows the resulting $E\left(k_{y}\right)$ subband dispersion for an ADQW. The dispersion $E\left(k_{x}\right)$ is not affected by the magnetic field, and $k_{x}$ is therefore set to zero in the diagram. The $E\left(k_{y}\right)$-dispersion curves of $|w 1\rangle$ and $|n 1\rangle$ intersect, for any $B_{x} \neq 0$, at a wave vector $k_{y}^{\text {res }}\left(B_{x}\right)$. Resonant tunneling with conservation of energy and transverse canonical momentum is possible at this intersection, and resonant transfer of electrons from $\mathrm{QW}_{\mathrm{n}}$ to $\mathrm{QW}_{\mathbf{w}}$ takes place if the states at $k_{y}^{\text {res }}\left(B_{x}\right)$ are occupied. After excitation, electrons cool to a temperature of $50 \mathrm{~K}$ within the time resolution [14], thus resonant transfer at low temperatures is only possible if $k_{y}^{\text {res }}\left(B_{x}\right)$ is at the subband edge of $|n 1\rangle$ (right-hand side inset of Fig. 2). The condition for resonant transfer from $\mathrm{QW}_{\mathrm{n}}$ to $\mathrm{QW} \mathrm{W}_{\mathrm{w}}$ is therefore

$\Delta E=E_{\mathrm{n} 1}-E_{\mathrm{w} 1}=\frac{\left(\hbar \Delta k_{y}\right)^{2}}{2 m^{*}}=\frac{\left(e \Delta z B_{x}\right)^{2}}{2 m^{*}}$.

Fig. 2 shows $\tau_{\mathrm{n}}$ versus $B_{1}$ for an ADQW (10 times repeated and isolated by $20 \mathrm{~nm} \mathrm{Al}_{0.35} \mathrm{Ga}_{0.65}$ As layers) with a $10 \mathrm{~nm}$ and a $5 \mathrm{~nm}$ QW separated by a $6 \mathrm{~nm}$ $\mathrm{Al}_{0.35} \mathrm{Ga}_{0.65} \mathrm{As}$ barrier. The luminescence decay time $\tau_{\mathrm{n}}$ of $\mathrm{QW}_{\mathrm{n}}$ exhibits a pronounced minimum around $16 \mathrm{~T}$. The resonance position agrees well with the theoretical value of $15 \mathrm{~T}$, calculated from Eq. (1). The luminescence decay time $\tau_{w}$ of $Q W_{w}$ does not vary significantly with $B_{\perp}$, confirming that the reduction in $\tau_{\mathbf{n}}$ is caused by the influence of $B_{\perp}$ on tunneling only and not on recombination. We do not observe any tunneling resonance between different Landau levels in a magnetic field $B_{\|}$in $z$-direction. The wave functions for different Landau levels are orthogonal, and therefore the Landau quantum number must be conserved in an ideal system, analogous to conservation of transversal momentum for $B_{||}=0$.

Resonant transfer from $\mathrm{QW}_{\mathrm{n}}$ to $\mathrm{QW}_{\mathrm{w}}$ is only observable, if the electrons are scattered out of the resonant states in $\mathrm{QW}_{\mathrm{w}}$. The resonant tunneling time is therefore determined by both the coupling through the barrier and an inter- or intrasubband scattering process in $\mathrm{QW}_{\mathrm{w}}$. The influence of the subband distance $\Delta E$ on the tunneling times provides valuable information on the dominant scattering process. We vary the subband distance $\Delta E$ by an electric field in $z$-direction. Decreasing $\Delta E$ shifts the resonance to lower magnetic fields. The dependence of the resonant decay times on $\Delta E$ is shown in Fig. 3. Resonant tunneling is faster as soon as the subband spacing exceeds the GaAs longitudinal optical (LO) phonon energy of $\hbar \omega_{\mathrm{LO}}=36 \mathrm{meV}$; the resonant decay

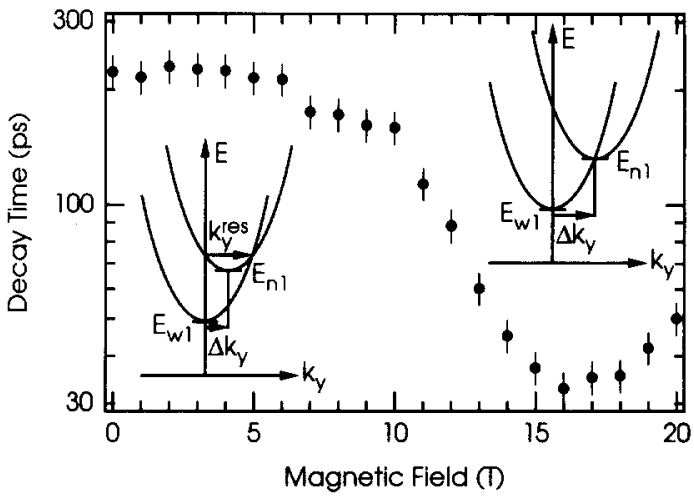

Fig. 2. Luminescence decay time $\tau_{n}$ of $Q W_{n}$ versus magnetic field perpendicular to growth direction. Inserts: $E\left(k_{y}\right)$-dispersion of the lowest electronic subbands $|w 1\rangle$ and $|n 1\rangle$ in an ADQW with magnetic field in the $x$-direction. The left diagram shows the situation where resonant transfer of electrons from $Q W_{n}$ to $\mathrm{QW}_{\mathrm{w}}$ is not possible at low temperatures. In the right diagram resonant transfer is possible.

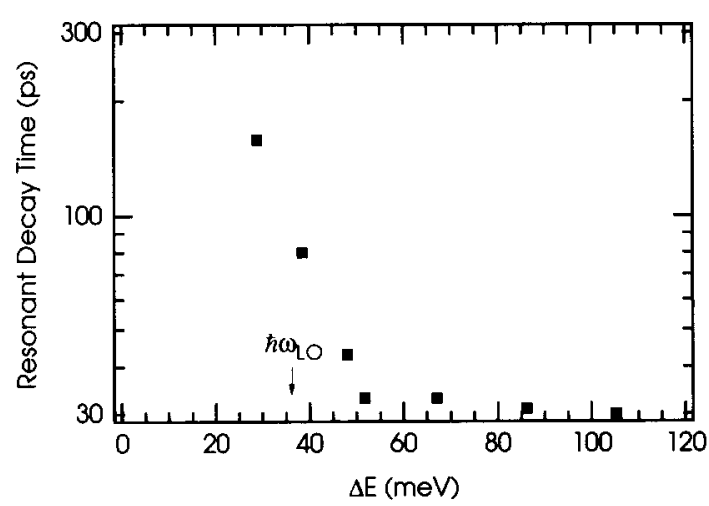

Fig. 3. Resonant luminescence decay time versus subband distance $\Delta E$.

times saturate for large $\Delta E$ at a minimum of $30 \mathrm{ps}$. This result shows that the transfer from $\mathrm{QW}_{\mathrm{n}}$ to $\mathrm{QW}_{\mathrm{w}}$ becomes only fast if the electrons are rapidly swept out of the coupled state in $\mathrm{QW}_{\mathrm{w}}$ by $\mathrm{LO}$ phonon emission. Earlier experiments showed that in absence of magnetic field, LO phonon participation is not required for efficient tunneling because abundant defect-assisted scattering prevails [12].

\section{Spin relaxation}

Absorption spectra recorded under a high magnetic field $B_{\| \mid}$in $z$-direction show discrete peaks associated with magnetoexcitons and a disappearance of features 
associated with the free carrier continuum. Such a modified energy spectrum changes the dynamics of magnetoexcitons, for instance, the thermalization processes. Moreover, magnetoexcitons are spin-split by the Zeeman effect due to their different orientation of magnetic moments of electrons and holes. The allowed optical transitions involve a change $\Delta M_{\mathrm{S}}= \pm 1$. Steady-state photoluminescence experiments indicated that the conservation of electron spin during the relaxation from the excited states to the 1s ground state is favored with increasing magnetic field and that the electron spin relaxation time between the two 1s heavy-hole magnetoexcitons with opposite electron spin becomes comparable to the excitonic radiative lifetime [5].

Excitonic states of well-defined electron spin can be excited selectively by adjusting the laser energy and polarization to a given magnetoexcitonic transition. The exciton dynamics including spin relaxation can then be clearly studied. We have investigated the spin-flip process measuring the photoluminescence decay times of excitons with different electron spin orientation. Magnetic momentum relaxation of holes is known to be much faster in comparison with electron spin relaxation, and an immediate thermalized population of the hole levels can be assumed in our experiment.

Our sample contains three $9 \mathrm{~nm} \mathrm{GaAsQWs}$ sandwiched between $100 \mathrm{~nm} \mathrm{Al}{ }_{0.26} \mathrm{Ga}_{0.74}$ As barriers, grown on an undoped (100) GaAs substrate. At zero magnetic field, the luminescence spectra of the QWs exhibit two transitions line width of 1-1.5 meV separated by $1 \mathrm{meV}$ [15]. The dependence of the relative intensities of both transitions on the excitation density shows that the high-energy transition is a free exciton decay and that the low-energy transition is caused by the radiative decay of localized excitons since the intensity of the latter saturates at high excitation densities. Four transitions - two pairs of two transitions with opposite polarizations - are resolved at magnetic fields $B_{\|} \geqslant 10 \mathrm{~T}$ (Fig. 4). Both highenergy transitions, labelled excitons $A$ and $B$, originate from free exciton decay. The low-energy transitions, labelled excitons $\mathrm{A}^{\prime}$ and $\mathrm{B}^{\prime}$, are the decays of localized excitons where $\mathrm{A}$ and $\mathrm{B}$ stands for $\sigma^{+}$and $\sigma^{-}$polarization, respectively. The energy difference between $A$ and $B$ corresponds to the combined Zeeman splitting of electrons and holes.

In quasi-two-dimensional GaAs layers the valence band is energetically split into a heavy-hole band at lower and a light-hole band at higher energy. An excitonic state is related with each of these valence bands. The high-energy exciton states can be only probed by photoluminescence excitation spectroscopy. We now discuss the different magnetic field regimes. For magnetic fields $B_{\|}>5 \mathrm{~T}$, the 1s heavy-hole and light-hole excitons are at lower energy than the $2 \mathrm{~s}, 3 \mathrm{~s}, \ldots$ states [5]. For

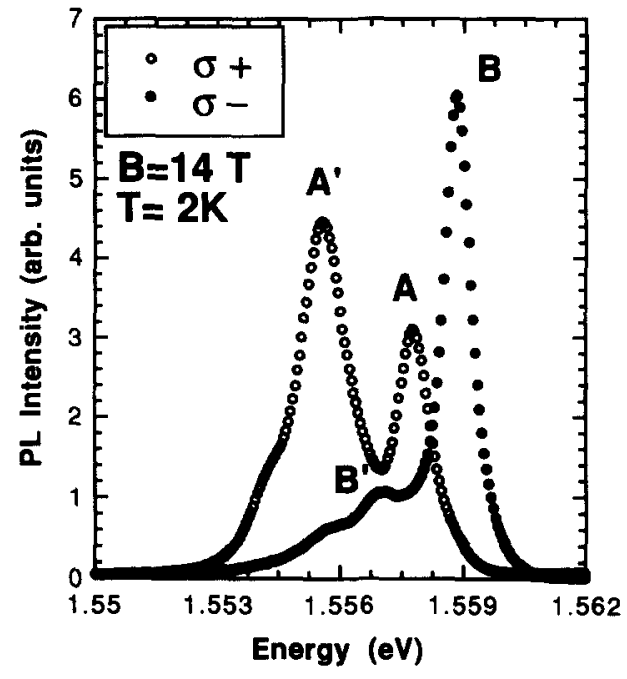

Fig. 4. Steady-state PL spectra at $B_{\|}=14 \mathrm{~T}$ (polarized $\sigma^{-}$and $\sigma^{+}$) under $\sigma$-excitation of the light-hole level. The four main transitions $\mathrm{B}, \mathrm{A}, \mathrm{B}^{\prime}$, and $\mathrm{A}^{\prime}$ are shown.

$B_{\|}>12 \mathrm{~T}$, the Zeeman splitting of the 1s heavy-hole transitions can be resolved, which show different circular polarizations according to the selection rule $\Delta M_{\mathrm{S}}= \pm 1$. The transitions of the 1s light-hole excitons show an even stronger Zeeman splitting. Selective excitation of lighthole excitons with specific electron spin orientation is therefore possible both, by photon energy or circular polarization of the laser.

First, we show results obtained with continuous wave (CW) excitation. Fig. 4 displays the $\sigma^{+}$and $\sigma^{-}$polarized spectra at $B_{\|}=14 \mathrm{~T}$ with $\sigma$-excitation of the $1 \mathrm{~s} \mathrm{light-hole}$ state. The most striking feature is the high intensity of the high-energy line $B$, which is roughly twice as high as the intensity of line A. This excessive intensity of line B is a strong indication for a nonthermal occupation of the Zeeman-split spin states. The population inversion increases with higher magnetic field, leading to the conclusion that electron spin relaxation is slower at higher magnetic fields [5]. However, time-resolved luminescence experiments reveal that this interpretation is wrong.

Fig. 5 shows the temporal variation of the various lines at (a) $21 \mathrm{~T}$ and (b) $14 \mathrm{~T}$. The excitation conditions are adjusted such that the time-integrated spectra look similar to the $\mathrm{CW}$ results. Once more the 1s light-hole exciton is excited with $\sigma$-light. The mean exciton density is about $10^{10} \mathrm{~cm}^{-2}$. Initially the $\mathrm{B}$ line dominates by more than an order of magnitude. After a rapid initial decay with a lifetime of $\tau_{1}=44 \mathrm{ps}$, the intensity of the $B$ line is lower than the intensity of the $\mathrm{A}$ line. Both lines show the same final decay time of $\tau_{2}=200 \mathrm{ps}$. The decay time $\tau_{1}$ 


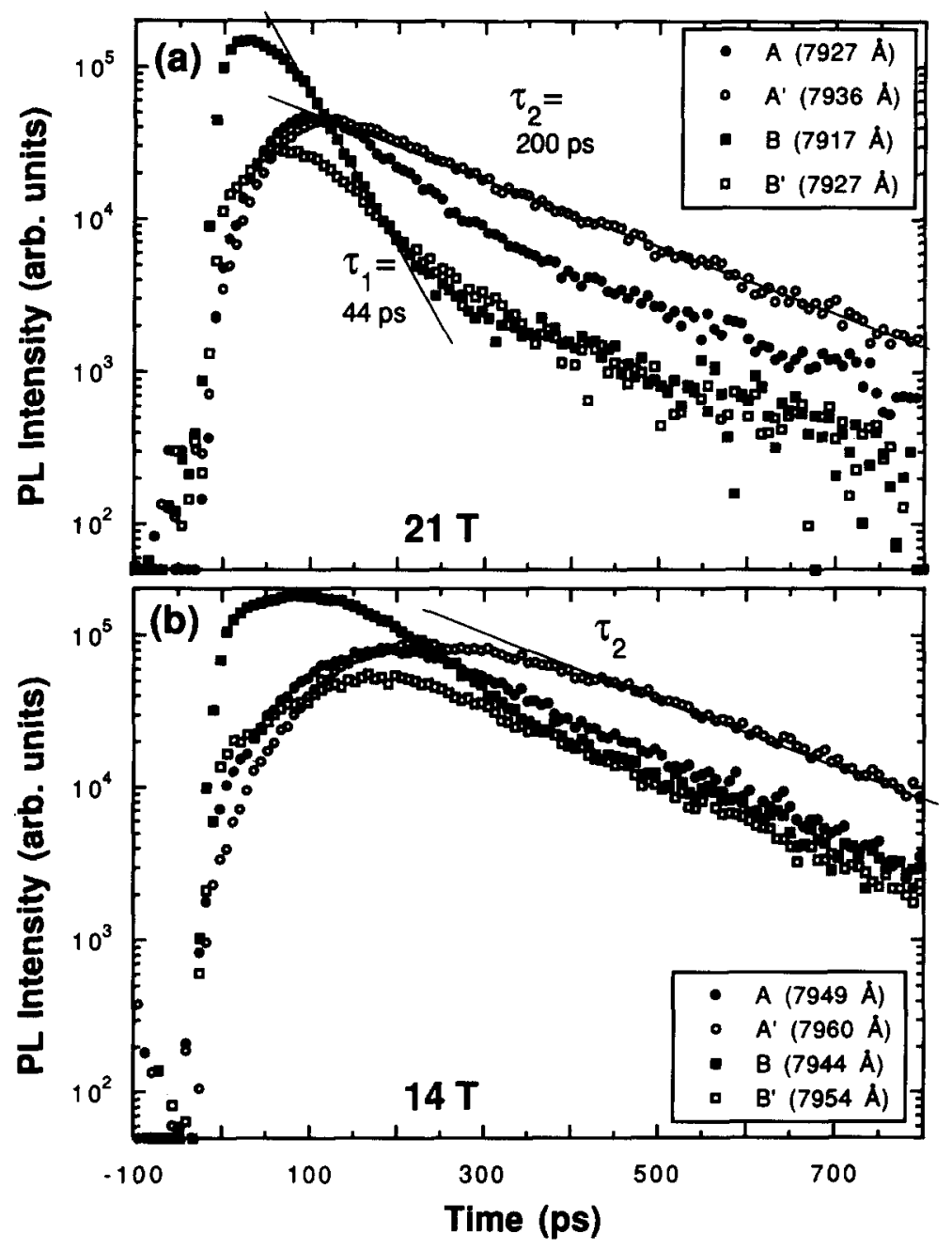

Fig. 5. Transients of the photoluminescence transitions $\mathbf{B}, \mathbf{A}, \mathbf{B}^{\prime}$, and $\mathbf{A}^{\prime}$ at $21 \mathrm{~T}$ (a) and $14 \mathrm{~T}$ (b) under $\sigma$-excitation of the light-hole level. Note the initial fast decay of the B line.

decreases with increasing magnetic field (compare Fig. 5(a) and (b)) whereas $\tau_{2}$ stays constant.

Simultaneously with a fast initial decay of line B, all other transitions, in particular line $\mathbf{A}$ and $\mathbf{A}^{\prime}$ involving free and localized exciton states with opposite spin polarization still increase in intensity with time. Obviously, the free exciton $B$ is initially generated very fast by direct relaxation from the light-hole to the heavy-hole exciton state with electron spin conservation. Afterwards, various relaxation channels into the $\mathbf{A}, \mathbf{A}^{\prime}$, and $\mathbf{B}^{\prime}$ lines involving spin-flip and localization processes give rise to the fast initial decay of the B line. At long delay times, after $400 \mathrm{ps,}$, all excitons decay with the same decay time $\tau_{2}$, indicating that the occupation of the different states reaches thermal equilibrium. A measure for the exciton relaxation time is obtained by plotting the ratio $B / A$ of the intensities of the $B$ and $A$ line as a function of time (see Fig. 6). This ratio is not directly the spin relaxation time since it includes transfer into localized states. However, Fig. 5 clearly shows that the initial loss of intensity of line $B$ is predominantly due to transfer of intensity into lines $A$ and $A^{\prime}$, i.e., a spin-flip relaxation. Obviously the exciton relaxation time including spin flip depends on magnetic field decreasing from $130 \mathrm{ps}$ at $12 \mathrm{~T}$ to $50 \mathrm{ps}$ at $21 \mathrm{~T}$. The times are compiled in Fig. 6(b). The ratio $I_{0} / I_{\infty}=(B / A)_{t=0} /(B / A)_{t \rightarrow \infty}$ is also shown. This value is a measure for the conservation of spin during the fast initial relaxation from light- into heavy-hole excitons. This spin conservation strongly increases with increasing magnetic field. 

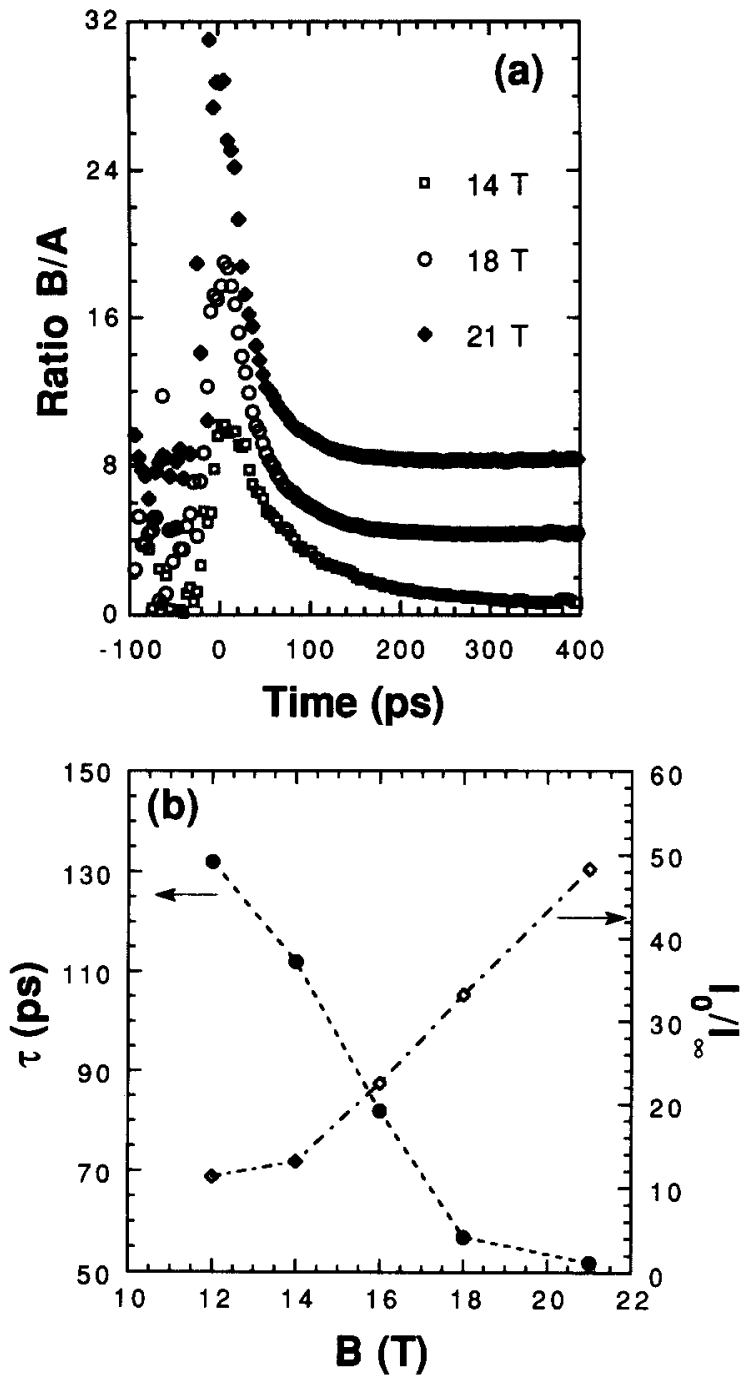

Fig. 6. (a) Time dependence of the intensity ratio $B / A$ at different values of the magnetic field. The transients can be approximated by an exponential decay with a lifetime $\tau$ which corresponds to the exciton relaxation time including spin flip. Note the decrease of $\tau$ but the increase of $B / A$ with increasing magnetic field. (b) Dependence of the exciton relaxation time $\tau$ including spin flip and of the excess population $I_{0} / I_{\infty}(t)$ upon magnetic field. The dashed and dashed-dotted lines are guides to the eye.

In summary, electron spin conservation during the initial light- to heavy-hole relaxation increases with magnetic field, and exciton relaxation afterwards including spin flip fastens with increasing magnetic field. Both effects contribute to the intensity ratio of the $B$ and A lines as observed in cw photoluminescence. Obviously, the magnetic field dependence of electron spin conserva- tion during the initial light- to heavy-hole relaxation prevails since the ratio $B / A$ in the $\mathrm{cw}$ experiment increases with magnetic field.

We want to point out that only the time-resolved experiments revealed that actually two different counteracting processes have a strong and different magnetic field dependence.

\section{Conclusions}

We demonstrated the strong influence of a high magnetic field on the carrier dynamics in quantum wells for the examples of tunneling and spin relaxation. A magnetic field $B_{\perp}$ can induce resonant tunneling which differs significantly from transfer without field. Exciton relaxation including spin flip is enhanced by a magnetic field $B_{\| \text {. }}$ The latter example shows that only with time-resolved experiments a clear identification of different magnetic field-dependent relaxation processes becomes possible.

\section{Acknowledgements}

We would like to use this opportunity to express our sincere appreciation to Professor Peter Wyder and his staff at the High-Field-Magnet laboratory in Grenoble for his continuous support to so many researchers utilizing the high fields available at this French-German joint facility.

\section{References}

[1] J.C. Maan, in: Festkörperprobleme (Vieweg, Braunschweig, 1987) Vol. 27, p. 137.

[2] C. Weisbuch and B. Vinter, Quantum Semiconductor Structures (Academic Press, Boston, 1991).

[3] G.E.W. Bauer and T. Ando, Phys. Rev. B 37 (1988) 3130, and references therein.

[4] A. Fasolino, G. Platero, M. Potemski, J.C. Maan, K. Ploog and G. Weimann, Surf. Sci. 267 (1992) 509.

[5] M. Potemski, J.C. Maan, A. Fasolino, K. Ploog and G. Weimann, Phys. Rev. Lett. 63 (1989) 2409.

[6] V. Langer, H. Stolz and W. von der Osten, Phys. Rev. Lett. 64 (1990) 854.

[7] J.B. Stark, W.H. Knox, D.S. Chemla, W. Schäfer, S Schmitt-Rink and C. Stafford, Phys. Rev. Lett. 65 (1990) 3033.

[8] R.T. Bate, Fut. El. Device J. 4 (Suppl. 1) (1993) 5.

[9] D.Y. Oberli, J. Shah, T.C. Damen, C.W. Tu, T.Y. Chang, D.A.B. Miller, J.E. Henry, R.F. Kopf, N. Sauer and A.E. DiGiovanni, Phys. Rev. B 40 (1989) 3028. 
[10] M.G.W. Alexander, M. Nido, W.W. Rühle and K. Köhler, Phys. Rev. B 41 (1990) 12295.

[11] T. Matsusue, M. Tsuchiya, J.N. Schulman and H. Sakaki, Phys. Rev. B 42 (1990) 5719.

[12] A.P. Heberle, W.W. Rühle, M.G.W. Alexander and K. Köhler, Semicond. Sci. Technol. B 7 (1992) 421.

[13] T. Ando, J. Phys. Soc. Japan. 39 (1975) 411.
[14] K. Leo, W.W. Rühle and K. Ploog, Phys. Rev. B 38 (1988) 1947.

[15] S. Haacke, A. Heberle, M. Potemski, J. Maan, W. Rühle and G. Weimann, in: Proc. 6th Internat. Conf. on Modulated Semiconductor Structures (Garmisch-Partenkirchen, Germany, 1993), accepted for publication in Solid State Electronics. 